\title{
Solid Internal Energy Dissipation of 3C Single-Crystal Silicon Carbide Micromechanical Resonators by Heterojunction Growth on Silicon
}

\author{
Wenteng Chang* \\ Deptartment of Electrical Engineering, National University of Kaohsiung, \\ No. 700, Kaohsiung University Road, Nan-Tzu District, Kaohsiung, Taiwan, 811
}

(Received March 4, 2009; accepted July 29, 2009)

Key words: resonator, silicon carbide, internal energy dissipation, heterojunction growth

A previous report shows that a 100 -nm-thick free-free beam, single-crystal silicon carbide $(\mathrm{SiC})$ resonator has quality factors 10 times lower than those made with a $10-\mu \mathrm{m}$-thick single-crystal $\mathrm{SiC}$ cantilever even though the free-free beam is supposed to have lower clamping loss. ${ }^{(1,2)}$ This manuscript explores the above-mentioned difference using the heterojunction growth of (110) 3C-silicon carbide deposited as structural films of micromechanical resonators on single-crystal silicon and polycrystalline silicon. The polycrystalline $\mathrm{SiC}$ resonators with smaller clamping losses were fabricated to contrast with the resonators made of single-crystal $\mathrm{SiC}$. The analysis showed that solid internal energy dissipation may play a more important role than the other losses operated in tens of kilohertz. The resonators with a 2- $\mu$ m-thick single-crystal $\mathrm{SiC}$ having $Q$ s between the previous reports may suggest a higher defect density near the interface, which causes high solid internal dissipation. Although an electrical measurement technique was used, the dominance of this material property appeared to be due to grain size instead of conductivity.

\section{Introduction}

Micro-electromechanical system (MEMS) resonators have attracted the radio frequency (RF) communications community's attention because of their high quality factors $(Q s)$ and their capacity to be integrated into silicon-based integrated circuits (ICs). ${ }^{(3-5)}$ In addition, RF applications demand a device technology that operates from very low to ultrahigh frequencies. Thus, silicon carbide $(\mathrm{SiC})$ is a promising material for RF MEMS because of its high Young's modulus-to-density ratio, which increases the acoustic velocity and fundamental resonant frequency of the vibrating structure above the $\mathrm{Si}$ film. Among the approximately 250 known polytypes of $\mathrm{SiC}, 3 \mathrm{C}-\mathrm{SiC}$ (or $\beta$-SiC) is the only polytype that can be epitaxially grown as a single-crystal film on silicon

"Corresponding author: e-mail: wtchang@nuk.edu.tw 
substrates owing to its common crystal structure. Hence, $3 \mathrm{C}-\mathrm{SiC}$ is the only $\mathrm{SiC}$ suitable for integration with the current silicon-based technology.

The inverse of quality factor $\left(Q^{-1}\right)$ is a common and simple way to express energy losses in an oscillating system. It is mainly defined as energy dissipated over the stored energy in a dynamic system in an oscillation cycle. Energy losses in a microscale resonator can be attributed to several factors, including ambient damping, anchor-based clamping losses, thermoelastic damping (TED), and internal friction of the structural material. ${ }^{(6,7)}$ The total energy loss in the system is simply calculated as the sum of the energy loss components and can be written as follows:

$$
Q_{\mathrm{total}}^{-1}=Q_{\mathrm{air}}^{-1}+Q_{\mathrm{TED}}^{-1}+Q_{\text {clamping }}^{-1}+Q_{\text {solid }}^{-1}+\ldots \ldots .
$$

In measuring the transmission coefficient of a resonator, its total energy loss $\left(Q^{-1}\right.$ total $)$ can be extracted directly from the resonant frequency divided by the bandwidth of 3 $\mathrm{dB}$, which dropped from its resonant frequency. However, the measured maximum $Q$ is limited by the TED or Akhieser effect. It is common knowledge that TED is dependent on resonant frequency, although material characteristics also determine the dissipation. The Akhieser effect is purely material-property-related. Although SiCbased micromachined resonators have been available for more than a decade, and are considered to comprise one of the highest achievable Q-multiplied-by-operating frequency materials ${ }^{(8)}$ only a handful of research studies have been able to characterize the energy loss mechanisms in these devices. An early effort by $\mathrm{Su}$ et al. focused on describing the $Q$ of micromachined cantilever-based resonators made from $\mathrm{SiC}$ films. ${ }^{(1)}$ Their work used micromachined cantilevers fabricated from (100) 3C-SiC films epitaxially grown on (100) Si substrates. These large cantilevers measured $1 \mathrm{~mm}$ by 3.5 $\mathrm{mm}$ by $10 \mu \mathrm{m}$ according to MEMS standards. They were also used to portray energy damping as a function of temperature. The mechanical energy dissipation was calculated from the decay in resonance amplitude of the electrostatically actuated cantilevers, and the cantilever motion was detected by a capacitive sensor. This research showed that the energy dissipation $\left(Q^{-1}\right)$ for the single-crystal $3 \mathrm{C}$-SiC films was approximately $8 \times 10^{-6}$, which translated to a $Q$ of about 125,000 . The authors attributed the energy dissipation entirely to the internal friction caused by crystalline defects. However, they were not able to discuss the cause of this defect further.

Several years later, the work of Su et al. was followed by that of Kuo et al., in which the $Q$ of $\mathrm{SiC}$ lateral resonators fabricated from thinner $3 \mathrm{C}-\mathrm{SiC}$ films was measured.(9) In the study of Kuo et al., the single-crystal $\mathrm{SiC}$ films were nearly a factor of 5 thinner $(2 \mu \mathrm{m})$, which placed them well within the accepted thickness range for surface micromachined MEMS devices. Moreover, they used conventional comb-drive lateral resonators as test devices to make the study more relevant to a wider range of application areas where such device designs are used. Similarly, they used a transimpedance amplifier-based actuation and detection method. They found that devices made of (100) oriented 3C-SiC had a $Q$ of approximately 103,000, whereas those made of (110) oriented 3C-SiC had about 76,000. Unfortunately, Kuo et al. were not able to evaluate 
the devices beyond the $Q$ determination. Aside from research performed in the 2 to 10 $\mu \mathrm{m}$ thickness range, the $Q$ of $3 \mathrm{C}-\mathrm{SiC}$ resonators was also determined on the $100 \mathrm{~nm}$ scale. At the same time, researchers at Caltech focused on developing $3 \mathrm{C}$-SiC nanoelectromechanical system (NEMS) resonators; in particular, they have been studying energy dissipation among these structures..$^{(2,10,11)}$ The group at Caltech has characterized energy dissipation in both clamped-clamped and free-free beam designs. In their study, $Q$ was measured by actuating the devices in a vacuum at cryogenic temperatures using the magnetomotive transduction technique. Even the free-free beam was expected to have one order of magnitude higher $Q$ than the clamped-clamped beam. ${ }^{(12)}$ Their results indicated that the effective $Q$ s of free-free beam devices have a maximum of 11,000 . Evidently, they performed an in-depth analysis of these devices and concluded that clamping losses and surface roughness can play a key role in energy dissipation.

Despite these findings, the large difference in $Q$ (more than approximately 10 times the difference) in previous experiments has never been explained, although all the experiments used single-crystal $\mathrm{SiC}$ as resonators. Nevertheless, the extrapolation of the findings from $10-\mu \mathrm{m}$ - and 100-nm-thick beams for devices in the $2 \mu \mathrm{m}$ thickness range may not be appropriate. This is because the defect density in the heteroepitaxial 3C-SiC is known to exhibit a significant gradient with respect to thickness from a cross-sectional transmission electron microscopy (TEM) image (Fig. 1). ${ }^{(13)}$ The defect density near the 3C-SiC/Si interface is extremely high; it is in fact too high to be quantified accurately. This region extends by about $100 \mathrm{~nm}$ from the interface, and the defect density decreases considerably as defects intersect above this region. These defects are mainly stacking faults and twins, which intersect and annihilate one another in the process. This effect has a thickness dependence that extends beyond $2 \mu \mathrm{m}$ from the interface. Consequently, a work using NEMS resonators is not relevant for surface-micromachined MEMS because the former is made of a material with latent defects, which would undoubtedly

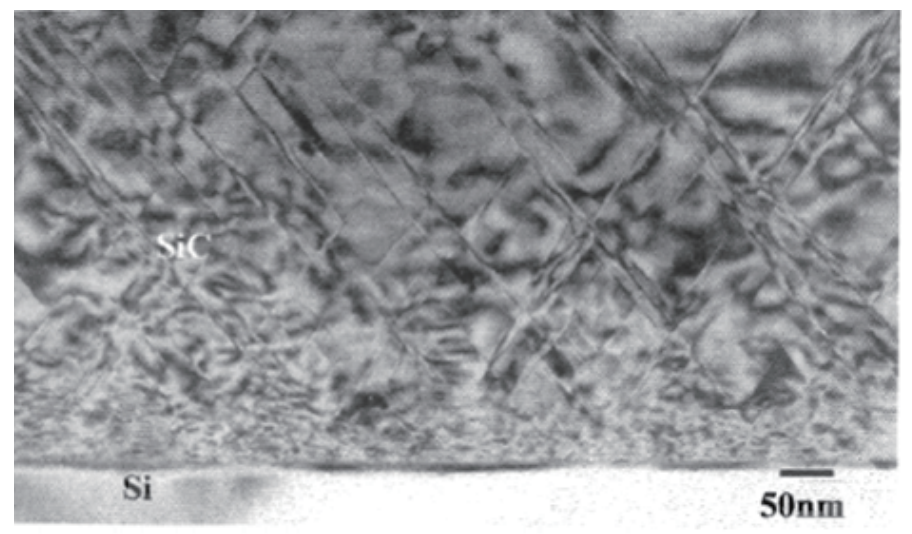

Fig. 1. Cross-sectional TEM image of a typical epitaxial 3C-SiC film. ${ }^{(13)}$ The defect density in heteroepitaxial $3 \mathrm{C}-\mathrm{SiC}$ exhibits a significant gradient with respect to thickness. 
skew the findings in a film with a relatively small thickness. Moreover, a smaller crystal within the interface can result in a higher solid internal energy dissipation. This is caused by the chemical bonds that are broken and reformed in an oscillating beam. ${ }^{(14)}$ Similarly, a previous study which used $10-\mu \mathrm{m}$-thick cantilevers may also be considered immaterial because it was performed using a material whose defect density was probably too low in the thickness range of interest $(2 \mu \mathrm{m})$. Thus, the research carried out by Kuo et al., which employed 2- $\mu \mathrm{m}$-thick resonators, provides a good impetus for further work. However, new measurements are required to obtain suitable data for in-depth analysis since those used in the previous work are noisy. ${ }^{(9)}$

In order to eliminate the clamping-damping of single-crystal $\mathrm{SiC}$ resonators, folded-beam resonators were designed; they were made of poly-SiC. This is because the difference in measured $Q$ between the single-crystal and poly-SiC resonators will become the difference in solid internal loss in accordance with eq. (1), especially if the air damping is negligible and the TED is calculable.

\section{Materials and Fabrication}

\subsection{Single-crystal $3 \mathrm{C}$-SiC resonators (Type $\mathrm{K}$ )}

A single-crystal $3 \mathrm{C}$-SiC film was grown using a heteroepitaxial process by atmospheric pressure chemical vapor deposition (APCVD). High-quality 3C-SiC films were found on Si substrates with void-free interfaces. The heteroepitaxial process is a three-step detailed growth procedure. ${ }^{(15)}$ However, heteroepitaxial $3 \mathrm{C}-\mathrm{SiC}$ films are not exactly suitable for the surface-micromachined lateral resonant-type device described in this study. This type cannot be electrically isolated from the underlying substrate because the device is situated directly on top of a $\mathrm{Si}$ wafer. To form a substrate suitable for surface micromachining, a film transfer technique called grow-a-substrate (GAS) was used. ${ }^{(9)}$ The process flow is shown in Fig. 2. In the following epitaxial growth using the above procedure, the $3 \mathrm{C}-\mathrm{SiC}$ surface was treated by chemical mechanical polishing (CMP) to form a uniform and smooth $\mathrm{SiC}$ surface. It was then followed by cleaning with Piranha and RCA solutions to remove surface contamination. Afterwards, a 1.7- $\mu$ m-thick $\mathrm{SiO}_{2}$ film was grown by initially depositing a polysilicon film by low-pressure chemical vapor deposition (LPCVD). This was followed by completely oxidizing the film by wet thermal oxidation. The wafer-thick polysilicon layer of approximately $600 \mu \mathrm{m}$ in thickness was then deposited to serve as the substrate. Consequently, the Si substrate was removed by wet chemical etching. Finally, a $3 \mathrm{C}-\mathrm{SiC}$ film was grown on the original $3 \mathrm{C}$-SiC layer using a homoepitaxial process at $1,280^{\circ} \mathrm{C}$. Except for the carbonization step, the same processing sequence was employed in the heteroepitaxial process. The resulting substrate is sometimes referred to as a silicon carbide-on-insulator ( $\mathrm{SiCOI)}$ substrate. The crystal orientation of the heteroepitaxially grown 3C-SiC is dependent on the $\mathrm{Si}$ substrate on which it was originally grown. This means that the 3C-SiC film will have various orientations. In this research, the (110) 3C-SiC-orientated SiCOI substrate was fabricated. The film resistivity was measured to be $40 \Omega$-cm by the four-point probe measurement. 
(a) SiC Deposition

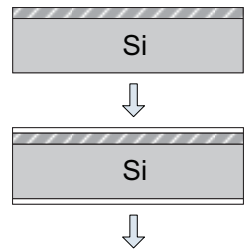

(b) $\mathrm{SiO}_{2}$ Deposition

Љ

(c) Thick Poly-Si Deposition

(d) Si Wet Etch

Poly-Si

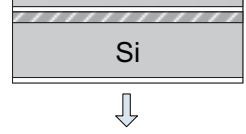

\&

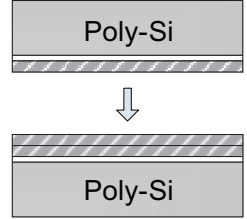

(e) SiC Regrowth

\section{Poly-Si}

Fig. 2. Cross-sectional process for forming single-crystal SiCOI substrates suitable for surface micromachining: (a) heteroepitaxial growth of $3 \mathrm{C}-\mathrm{SiC}$ on $\mathrm{Si}$; (b) formation of $\mathrm{SiO}_{2}$ as an electrically insulating sacrificial layer; (c) growth of a wafer-thick polysilicon film to serve as a supporting substrate; (d) removal of the silicon substrate by wet chemical etching; and (e) homoepitaxial 3C-SiC film regrowth.

The one-mask process was used in the fabrication sequence to define the $\mathrm{SiC}$ resonators. ${ }^{(9)}$ In this design, the buried $\mathrm{SiO}_{2}$ film of the $\mathrm{SiCOI}$ wafers was employed as a sacrificial layer and for facilitating substrate electrical isolation. The fabrication process began by depositing an $\mathrm{Al}$ thin film through magnetron sputtering. This film was patterned into an etch mask through photolithography, which basically defined the resonator. Afterwards, the $3 \mathrm{C}$-SiC film was anisotropically dry-etched using a reactive ion etching process based on $\mathrm{CHF}_{3}, \mathrm{O}_{2}$, and He. After the wafers were etched and diced, the chip level devices containing the resonators were treated with either an $\mathrm{Al}$ etchant or a Piranha solution to remove the Al mask. The structures were then released by wet etching in $49 \% \mathrm{HF}$ for $5 \mathrm{~min}$. The single-crystal 3C-SiC resonators used in this study were originally fabricated and denoted as Type K resonators. Figure 3(a) shows an overview scanning electron microscopy (SEM) image of a released folded-beam resonator that was fabricated from a single-crystal 3C-SiC film.

\subsection{Polycrystalline $3 C$-SiC resonators (Type $R$ )}

In this case, the poly-SiC growth procedure was identical to that used to grow epitaxial single-crystal 3C-SiC films, except that the substrate used here was polysilicon instead of single-crystal silicon. ${ }^{(16,17)}$ On the other hand, the substrate was a $1.5-\mu \mathrm{m}$ thick, thermally grown $\mathrm{SiO}_{2}$ film on (100) $\mathrm{Si}$ substrates. Prior to $\mathrm{SiC}$ growth, a 2- $\mu \mathrm{m}-$ thick polysilicon film was deposited on $\mathrm{SiO}_{2}$ film through LPCVD. This process is silane-based, which is the standard for polysilicon MEMS. After polysilicon deposition, 

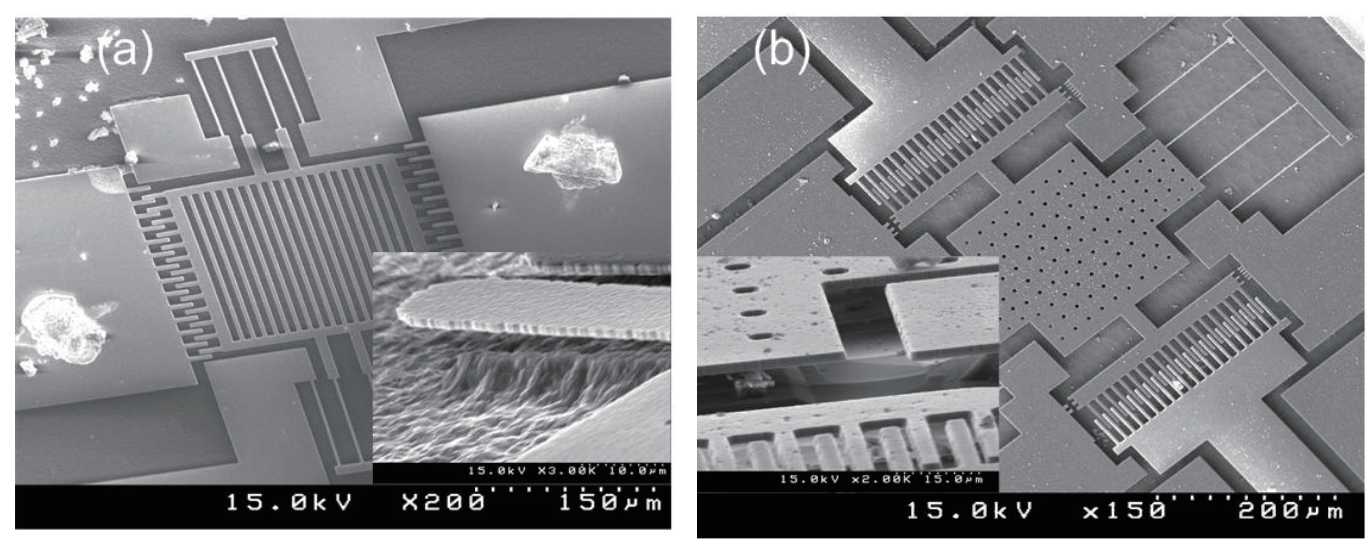

Fig. 3. (a) Overview SEM image of folded-beam resonators fabricated from single-crystal $3 \mathrm{C}-\mathrm{SiC}$ and (b) polycrystalline $3 \mathrm{C}-\mathrm{SiC}$.

the poly-SiC films were then deposited by APCVD. Similarly to single-crystal films, the poly-SiC films were unintentionally doped with residual nitrogen at levels considered sufficient to support electrostatic actuation. The films had a resistivity of $6.62 \Omega-\mathrm{cm}$, whereas the poly-SiC resonators were denoted as Type $\mathrm{R}$ resonators. Furthermore, polysilicon was used for both anchoring and sacrificial layers (Fig. 3(b)). Table 1 shows a summary of the fabrication details of the two devices. Table 1 shows a summary of the fabrication details of the two devices.

\section{Measurements}

The work employed electrical transmission to evaluate MEMS resonators. First, the chip-based MEMS resonators were mounted on a printed circuit board and a Philips SA5211 transimpedance amplifier with a gain of $14 \mathrm{k} \Omega$. During oscillation, the MEMS resonator generated a motional current converted to a voltage signal by the transimpedance amplifier. The transimpedance amplifier was chosen because of its bipolar junction transistor differential amplifier capable of producing a low-noise output signal. The test was conducted at $30 \mu$ Torr. The schematic diagram of the setup is detailed elsewhere. ${ }^{(18)}$ The motional current from the MEMS resonator was amplified by the transimpedance amplifier placed next to the MEMS chip. On the other hand, the transmission spectrum $\left(\mathrm{S}_{21}\right)$ determined the total $Q$ of the circuit containing the MEMS resonator. Subsequently, the circuit was placed in a vacuum system equipped with a diffusion pump, which may reach pressures of approximately $1 \times 10^{-6}$ Torr. Figure 4 shows a magnitude plot for Devices K1 and R1, which were tested at $30 \mu$ Torr and then measured using an Agilent 4395A network analyzer. This equipment was likewise used to determine the resonant frequency and $Q$ of this device. Note that each vertical grid in 
Table 1

Summary of fabrication details of the $3 \mathrm{C}-\mathrm{SiC}$ resonators evaluated in this study.

\begin{tabular}{lcc}
\hline & Type $\mathrm{K}$ & Type R \\
\hline Growth method & APCVD \\
SiC dep. temp. $\left({ }^{\circ} \mathrm{C}\right)$ & \multicolumn{2}{c}{1280} \\
Precursor gases & \multicolumn{2}{c}{$\mathrm{SiH}_{4}$ and $\mathrm{C}_{3} \mathrm{H}_{8}$} \\
Doping gas & None \\
SiC thickness $(\mu \mathrm{m})$ & 2 \\
Orientation & \multicolumn{2}{c}{$(110)$} \\
Substrate & Polysilicon & $\mathrm{SiO}_{2} /(100) \mathrm{Si}$ \\
Sacrificial/anchor & $\mathrm{SiO}_{2}$ & Polysilicon \\
\hline
\end{tabular}

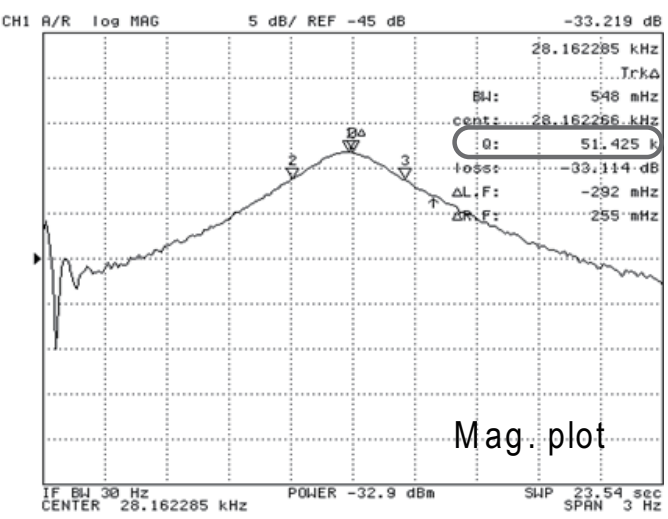

(a)

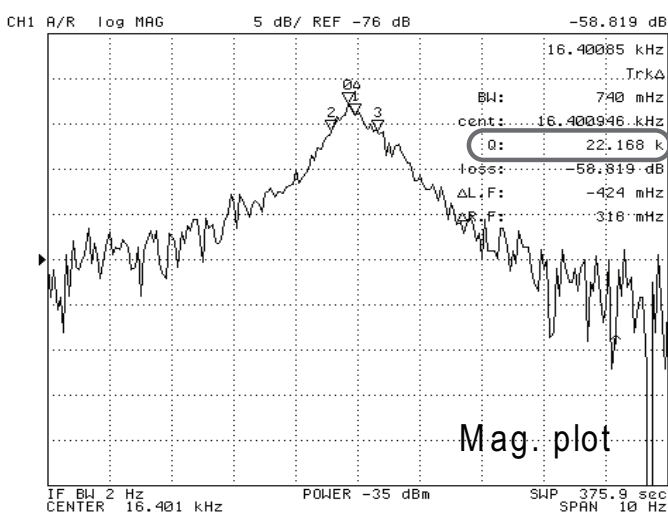

(b)

Fig. 4. (a) Magnitude plot for $\mathrm{K} 1$ single-crystal $\mathrm{SiC}$ resonator and (b) R1 polycrystalline $\mathrm{SiC}$ by APCVD. Their quality factors are 51,425 and 22,168, respectively.

the magnitude plot represents $5 \mathrm{~dB}$. The measured $Q \mathrm{~s}$ are listed in Table 2. The singlecrystal 3C-SiC resonators (K1 and $\mathrm{K} 4$ ) have $Q$ s of about 50,000 (Fig. 4(a)), while R1, which is also made from APCVD films, has a $Q$ of approximately 20,000 (Fig. 4(b)).

\section{Discussion}

\subsection{Air damping}

The air damping of flexural resonators can be rendered negligible if the ambient pressure is reduced to a critical point. ${ }^{(19,20)}$ In this study, the measurement indicates that the critical point for the folded-beam resonator design is about 10 mTorr. Although air damping is a very significant component of total energy dissipation, it is considered negligible if testing is performed under high-vacuum conditions. These findings indicate 
Table 2

Summary of the measured resonators.

\begin{tabular}{ccc}
\hline Device & $\begin{array}{c}\text { Resonant Freq. (Hz) } \\
\text { Ave. (Std. Dev.) }\end{array}$ & $\begin{array}{c}\text { Quality Factor } \\
\text { Ave. (Std. Dev.) }\end{array}$ \\
\hline K1 & $28162(0.5)$ & $49979(2420)$ \\
K4 & $16442(0.5)$ & $45947(2817)$ \\
R1 & $16399(1.9)$ & $20086(2914)$ \\
\hline
\end{tabular}

that for specific resonator designs in this study, the devices should be tested at pressures below 10 mTorr, which is considered to be well above the base pressure of the vacuum testing system.

\subsection{Thermoelastic damping (TED)}

In an oscillating flexural beam resonator, the relaxation rate of the bending beams affects TED. ${ }^{(10)}$ Using known material parameters for $3 \mathrm{C}-\mathrm{SiC}^{(21,22)}$ and the device dimensions measured from various SEM images, an estimate of the minimal energy dissipation due to TED was calculated using eqs. (2) and (3):(22)

$$
\begin{gathered}
Q_{\mathrm{TED}}^{-1}=\frac{\alpha^{2} T E}{\rho C_{\mathrm{P}}} \cdot \frac{\left(F / F_{0}\right)}{1+\left(F / F_{0}\right)^{2}} \sim \frac{\alpha^{2} T E}{\rho C_{\mathrm{P}}} \cdot \frac{F}{F_{0}} \text { if } F<<F_{0}, \\
F_{0}=\frac{\pi \kappa}{2 \rho C_{\mathrm{P}} t^{2}},
\end{gathered}
$$

where $C_{\mathrm{P}}$ is the heat capacity of the structural material, that is, $\mathrm{SiC}$ at constant pressure; $\alpha$ is the thermal expansion coefficient of the structural material; $T$ is the spatially mean temperature of the beam; $E$ is the elastic modulus of the structural material; $F$ is the resonant frequency; $F_{0}$ is the characteristic damping frequency; $\kappa$ is the thermal conductivity of the structural material; $\rho$ is the mass density of the structural material; and $t$ is the beam thickness. For a $2-\mu \mathrm{m}$ oscillation beam, the $F_{0}$ is about $47 \mathrm{MHz}$, which is far above the operating frequency in this work. The $Q^{-1}$ TED of $\mathrm{K} 1$ is thus plotted in Fig. 5 , which shows an order of $10^{-7}$. The manual calculations of $Q^{-1}$ TED for $\mathrm{K} 1$ and $\mathrm{R} 1$ are summarized in Table 3. For the single-crystal $3 \mathrm{C}-\mathrm{SiC}$ resonators, the energy dissipation specifically associated with TED is only 0.016 of the total. Even though the value is small, it is not considered insignificant. On the other hand, the energy dissipation of poly-SiC associated with TED is between 0.02 and 0.08 . It should be noted that the TED calculations for poly-SiC microstructures may lack accuracy. This is mainly because some key material properties, such as thermal expansion coefficient, heat capacity and thermal conductivity, are not specifically known for the microstructure and orientation of the poly-SiC films used in this study. ${ }^{(21)}$ Nonetheless, these calculations have been included to illustrate that the contribution of TED to the total energy dissipation can be considered comparable to a single-crystal 3C-SiC film even for poly-SiC. Thus, this 


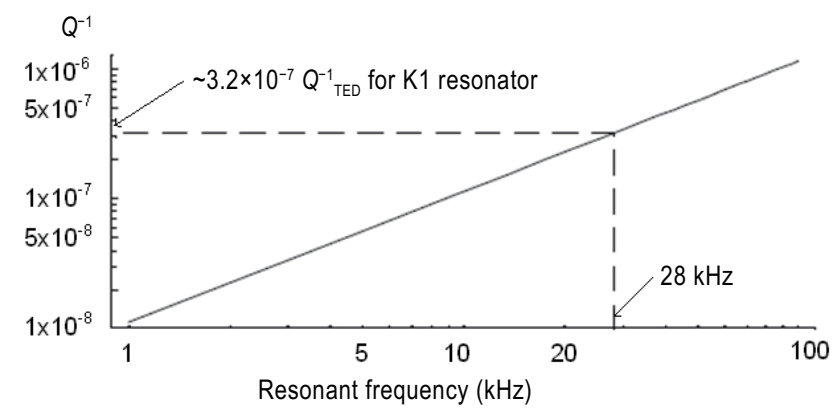

Fig. 5. The $Q^{-1}$ TED plot for $\mathrm{K} 1$ resonator.

Table 3

Summary of key material properties and required device parameters for calculating the contribution of TED to energy dissipation in SiC-based lateral resonators.

\begin{tabular}{lcc}
\hline & Type K & Type R \\
\hline Density $\left(\mathrm{kg} / \mathrm{m}^{3}\right)$ & \multicolumn{2}{c}{$3.21 \times 10^{3}$} \\
Heat Capacity $(\mathrm{J} / \mathrm{kg}-\mathrm{K})$ & $1.3 \times 10^{3}$ & $5.9 \times 10^{2} \sim 7.0 \times 10^{2(21)}$ \\
Thermal Expansion $(1 / \mathrm{K})$ & $4.2 \times 10^{-6}$ & $\sim 2.9 \times 10^{-6(22)}$ \\
Temperature $(\mathrm{K})$ & \multicolumn{2}{c}{$3.0 \times 10^{2}$} \\
Thermal Conductivity $(\mathrm{W} / \mathrm{m}-\mathrm{K})$ & $5.0 \times 10^{2}$ & $8 \times 10^{1} \sim 1.8 \times 10^{2(21)}$ \\
Beam Thickness $\left(\times 10^{-6}\right)$ & 2.2 & 1.9 \\
Resonant Frequency $(\mathrm{Hz})$ & $2.8160 \times 10^{4}$ & $1.6400 \times 10^{4}$ \\
$Q^{-1}{ }_{\text {TED }} @$ Resonant Frequency & $3.24 \times 10^{-7}$ & $1.26 \sim 4.03 \times 10^{-6}$ \\
$Q^{-1}{ }_{\text {total }}$ & $2.0 \times 10^{-5}$ & $5.0 \times 10^{-5}$ \\
$Q^{-1}{ }_{\text {TED }} / Q_{\text {total }}^{-1}$ & $1.63 \times 10^{-2}$ & $2.0 \times 10^{-2} \sim 8.0 \times 10^{-2}$ \\
\hline
\end{tabular}

alone cannot explain the large difference in $Q$. The range of $Q^{-1}{ }_{\mathrm{TED}} / Q^{-1}$ total values for Type $\mathrm{R}$ is due to the uncertainty parameters of heat capacity and thermal conductivity for a noncrystal structure.

\subsection{Clamping loss}

Generally, clamping losses for flexural resonators are strongly related to device design. ${ }^{(23)}$ Both the Type $\mathrm{K}$ and Type $\mathrm{R}$ devices used in this study were fabricated using the folded-beam design in order to eliminate clamping losses (Fig. 3). Both of the displacements of the resonators' trusses are about half of the proof masses owing to the folded-beam design (Fig. 6), in which red signifies the maximum displacement and blue signifies the minimum. The oscillation modeling shows that both types have the same oscillation modeling. Therefore, clamping loss is proportional to the cubic ratio of effective clamping length/width, that is,

$$
Q_{\text {clamping }}^{-1}=\beta\left(w_{\text {eff }} / l_{\text {eff }}\right)^{3},
$$



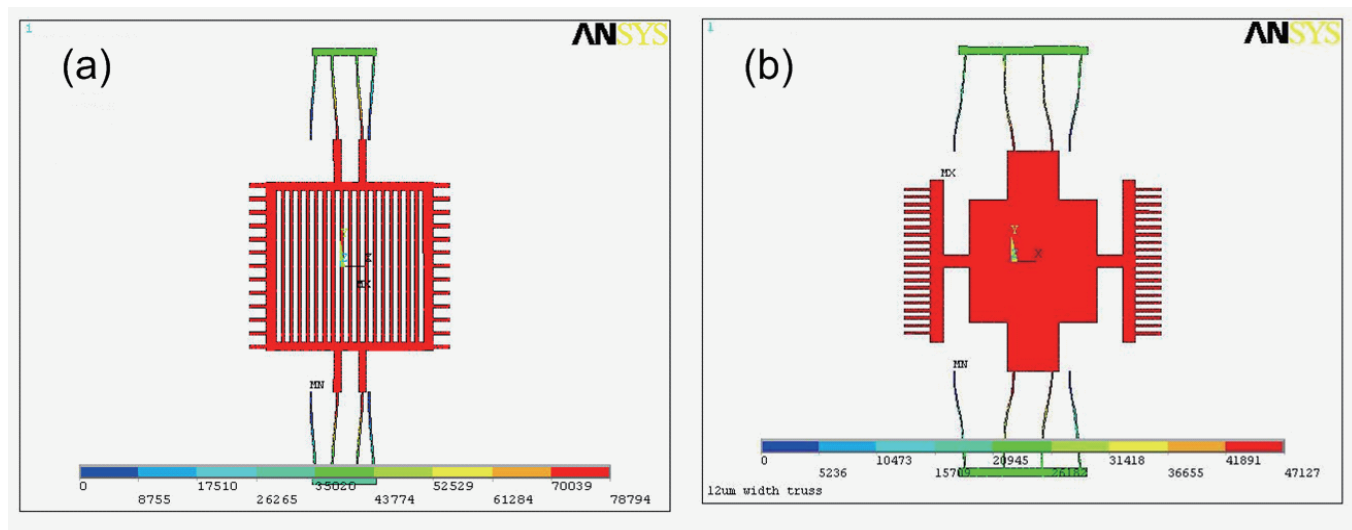

Fig. 6. Oscillation modeling of $\mathrm{K} 1$ and $\mathrm{R} 1$, showing the displacement of the trusses is half than that of the proof masses.

where $w_{\text {eff }}$ and $l_{\text {eff }}$ are the effective width and length of the beams, respectively; and the coefficient $\beta$ depends on the design of the resonator. ${ }^{(11,24)}$ The dimensions of the effective beams may cover the effective proof mass in the beams. Table 4 lists the dimensions of K1 and R1. Although K1 has strip etching holes, while R1 has circle etching holes, the dimensions of the proof masses are basically the same. The clamping losses are beam-width-sensitive according to eq. (4). The preliminary calculation shows that K1 has clamping losses about five times that of $\mathrm{R} 1$, assuming that the coefficients $\beta$ for $\mathrm{K} 1$ and $\mathrm{R} 1$ are the same. This assumption leads to $Q^{-1}$ clamping, $\mathrm{R}$ and $Q^{-1}$ clamping, $\mathrm{K}$ being smaller than $4.6 \times 10^{-6}$ and $9.2 \times 10^{-7}$, respectively, because the folded-beam resonators ideally have coefficients $\beta$ lower than 0.46 , as reported on cantilever beam resonators. ${ }^{(24)}$ In the work of Su et al., in which they used cantilever beam resonators, they found that these resonators have intuitively higher clamping losses $\left(Q^{-1}\right.$ clamping $)$ and $\beta$ than those of foldedbeam resonators used in this work, (24) although the overall $Q^{-1}$ total had a higher value. This indicates that clamping loss may not be a dominant factor in single-crystal resonators.

\subsection{Solid internal loss}

Owing to the geometric similarity of the aforementioned two devices, the $Q^{-1}$ total difference is rewritten as

$$
Q_{\text {total } \mathrm{R}}^{-1}-Q_{\text {total, } \mathrm{K}}^{-1}=\left(Q_{\text {solid, } \mathrm{R}}^{-1}-Q_{\text {solid, } \mathrm{K}}^{-1}\right)+\left(Q_{\text {clamping, } \mathrm{R}}^{-1}-Q_{\text {clamping, } \mathrm{K}}^{-1}\right)+\left(Q_{\mathrm{TED}, \mathrm{R}}^{-1}-Q_{\mathrm{TED}, \mathrm{K}}^{-1}\right) .
$$

The difference in total energy dissipation $\left(Q^{-1}{ }_{\text {total }}\right)$ is about $3 \times 10^{-5}$. However, the analysis indicates that $\mathrm{K} 1$ has clamping losses five times that of R1. Although $Q^{-1}$ TED,R was higher than $Q^{-1}$ TED,K , the order of $Q^{-1}$ TED was calculated to be two orders smaller than $Q^{-1}$ total for both $\mathrm{K} 1$ and $\mathrm{R} 1$. This implies that solid internal loss is the major energy loss for flexural-mode SiC resonators. The resonator's solid internal loss is entirely related 
Table 4

Dimensions of K1 and R1.

\begin{tabular}{lcc}
\hline Dimensions & $\mathrm{K} 1$ & $\mathrm{R} 1$ \\
\hline Beam Width $(\mathrm{m})$ & $2.2 \times 10^{-6}$ & $1.9 \times 10^{-6}$ \\
Beam Length $(\mathrm{m})$ & $1.0 \times 10^{-4}$ & $1.5 \times 10^{-4}$ \\
Proof Mass Length $(\mathrm{m})$ & $1.9 \times 10^{-4}$ & $1.9 \times 10^{-4^{*}}$ \\
Proof Mass Width $(\mathrm{m})$ & $2.0 \times 10^{-4}$ & $2.0 \times 10^{-4}$ \\
Proof Mass Area ${ }^{*}\left(\mathrm{~m}^{2}\right)$ & $2.48 \times 10^{-8}$ & $5.0 \times 10^{-8}$ \\
Truss Area $\left(\mathrm{m}^{2}\right)$ & $8.0 \times 10^{-10}$ & $1.2 \times 10^{-9}$ \\
\hline
\end{tabular}

* the upper and lower area parts are excluded

to material properties. ${ }^{(5,25)}$ The principal difference between the single-crystal and polycrystalline samples in this study is the microstructure. This is specifically true with regard to grain size, because the devices share a common chemical structure and unit cell (cubic $\mathrm{SiC}$ ). To examine the grain size of the $\mathrm{SiC}$ films, an X-ray diffraction analysis was performed. ${ }^{(13,27,28)}$ The average crystallite size $D_{\mathrm{hkl}}$ was determined from the main diffraction peak using ${ }^{(28)}$

$$
D_{\mathrm{hkl}}=\mathrm{k} \lambda /\left(\beta_{1 / 2} \cos \theta\right),
$$

where $\mathrm{k}$ is a constant (0.09) and $\lambda$ is the wavelength of $\mathrm{CuK}_{\alpha 1}$ radiation $(1.54 \AA)$. The broadening $\beta_{1 / 2}$ in the peak integral was corrected by subtracting the instrumental broadening using

$$
\beta_{1 / 2}=\sqrt{F W H M_{\text {sample }}^{2}-F W H M_{\text {standard }}^{2}} .
$$

Crystalline $\mathrm{Al}_{2} \mathrm{O}_{3}$ was used as a calibration standard. The standard full-width halfmaximum (FWHM) of the (110) $\mathrm{Al}_{2} \mathrm{O}_{3}$ peaks was 0.1348 . Using eqs. (6) and (7), the average crystallite sizes were calculated. The results are listed in Table 5.

\subsection{Electrical resistivity of SiC versus quality factor}

The analysis suggests that the measured differences are related to the differences in solid internal losses. The film resistivities of Type $\mathrm{K}$ and Type $\mathrm{R}$ are $40 \Omega$-cm and $6.62 \Omega-\mathrm{cm},{ }^{(8,17)}$ respectively. This indicates that Type $\mathrm{K}$ has a higher crystallization and electrical resistivity than Type $\mathrm{R}$, as determined by electrical measurement. This result agrees with the previous result, suggesting that the quality factor decreases as electrical resistivity decreases, since a higher doping concentration can lower SiC crystallization, and thus lead to a smaller grain size.(29) In other words, the electrical loss is not a dominant factor in determining quality factor. This, despite the fact that the $Q$ s were obtained by electrical measurement throughout the measurement. 
Table 5

Roughnesses and calculated grain sizes of Type K and Type R.

\begin{tabular}{lcc}
\hline & Type K [26] & Type R [27] \\
\hline Roughness (nm) & $27[13]$ & $25[27]$ \\
(110) SiC at 2 theta (degree) & \multicolumn{2}{c}{$60.045^{\circ}$} \\
FWHM (degree) & 0.141 & 0.277 \\
Broadening & 0.04 & 0.24 \\
Grain size (nm) & 384.60 & 65.72 \\
\hline
\end{tabular}

\section{Conclusions}

The growth substrate is important because it can develop into single-crystal and polycrystalline $\mathrm{SiC}$ 's. It is not surprising that single-crystal $3 \mathrm{C}-\mathrm{SiC}$ has a higher $Q$ than polycrystalline $3 \mathrm{C}-\mathrm{SiC}$. However, the thickness of single-crystal $\mathrm{SiC}$ resonators may be another highly important factor for $Q \mathrm{~s}$, particularly in the range within micrometers and below. This is because a higher defect density near the interface can result in a high internal solid dissipation. This solid internal energy dissipation could dominate the material property, which appears to be determined by grain size. The measurement in this work of putting 2- $\mu$ m-thick rendered $Q$ s between previous works suggests that the thickness of single-crystal silicon carbide micromechanical resonators achieved through heterojunction growth on silicon should be considered in evaluating $Q$ s.

\section{Acknowledgments}

The author wishes to thank C. Zorman and M. Mehregany for their helpful comments, as well as H. I. Kuo and S. Roy for the fabrication of the resonators.

\section{References}

1 C. M. Su, M. Wuttig, A. Fekade and M. Spencer: J. Appl. Phys. 77 (1995) 5611.

2 X. M. H. Huang, X. L. Feng, C. A. Zorman, M. Mehregany and M. L. Roukes: New J. Phys. 7 (2005) 1 .

3 C. T.-C. Nguyen and R. T. Howe: IEEE J. Sol. Sta. Cir. 34 (1999) 440.

4 J. F. Gong, Z. Y. Xiao and P. C. H. Chan: J. Micromech. Microeng. 17 (2007) 20.

5 A. Uranga, J. Teva, J. Verd, J. L. López, F. Torres, J. Esteve, G. Abadal, F. Pérez-Murano and N. Barniol: Electr. Lett. 41 (2005) 1327.

6 J. Yang, T. Ono and M. Esashi: J. Microelectromech. Syst. 11 (2002) 775.

7 P. Mohanty, D. A. Harrington, K. L. Ekinci, Y. T. Yang, M. J. Murphy and M. L. Roukes: Phys. Rev. B 66 (2002) 085416

8 S. A. Chandorkar, M. Agarwal, R. Melamud, R. N. Candler, K. E. Goodson and T. W. Kenny: IEEE MEMS 21st Int. Conf. (MEMS 2008) p. 74.

9 H. I. Kuo, C. A. Zorman and M. Mehregany: Dig. Tech. Papers 12th Int. Conf. Solid-State Sensors and Actuators (IEEE, Boston, 2003) p. 742.

10 R. Lifshitz and M. L. Roukes: Phys. Rev. B 61 (2000) 5600. 
11 X. L. Feng, C. A. Zorman, M. Mehregany and M. L. Roukes: Solid-State Sens., Actu. and Microsyst. Workshop (IEEE, Head Island, 2006) p. 86.

12 K. Wang, A. C. Wong and C. T. C. Nguyen: Microelectromech. Syst. J. 9 (2000) 347.

13 C. H. Wu: PhD Thesis, Case Western Reserve University (2001).

14 D. Heinen, H. G. Bohn and W. Schilling: J. Appl. Phys. 78 (1995) 893.

15 C. A. Zorman, A. J. Fleischman, A. S. Dewa, M. Mehregany, C. Jacob, S. Nishino and P. Pirouz: J. Appl. Phys. 78 (1995) 5136.

16 C. A. Zorman, S. Roy, C. H. Wu, A. J. Fleischman and M. Mehregany: J. Mater. Res. 13 (1998) 406.

17 S. Roy, R. G. DeAnna, C. A. Zorman and M. Mehregany: IEEE Trans. Elect. Dev. 49 (2003) 2323.

18 W. T. Chang, M. Mehregany and C. Zorman: Proc. 2nd IEEE Int. Conf. Nano/Micro Eng. and Mol. Syst. (IEEE, Bangkok, 2007) p. 740.

19 F. R. Blom, S. Bouwstra, M. Elwenspoek and J. H. J. Fluitman: J. Vac. Sci. Technol. B 1 (1992) 19.

20 C. J. Welham, J. Greenwood and M. M. Bertioli: Sens. Actuators, A 76 (1999) 298.

21 A. F. Pojur, B. Yates and B. T. Kelly: J. Phys. D Appl. Phys. 5 (1972) 1321.

22 T. V. Roszhart: Tech. Dig., 4th Solid-State Sens. and Actu. Workshop (IEEE, Head Island, 1990) p. 13.

23 W. T. Hsu, J. R. Clark and C. T. C. Nguyen: Proc. 11th Int. Conf. Solid-State Sens. and Actu. (IEEE, Munich, 2001) p. 1110.

24 Y. Jimbo and K. Itao: J. Horolog. Inst. Japan 47 (1968) 1.

25 D. Heinen, H. G. Bohn and W. Schilling: J. Appl. Phys. 78 (1995) 893.

26 H. I. Kuo: PhD Thesis, Case Western Reserve University 2002.

27 S. Roy: PhD Thesis, Case Western Reserve University 2001.

28 P. Joubert, B. Loisel, Y. Chouan: J. Electrochem. Soc. 134 (1987) 2541.

29 W. T. Chang and C. Zorman: Microsyst. Technol. 15 (2009) 875. 